\title{
Inhibition of endothelial cell survival and angiogenesis by protein kinase $A$
}

\author{
Semi Kim, Manjiri Bakre, Hong Yin, and Judith A. Varner \\ University of California, San Diego Comprehensive Cancer Center, La Jolla, California, USA
}

\begin{abstract}
Receptors for the provisional ECM are important regulators of angiogenesis. One of these receptors, integrin $\alpha 5 \beta 1$, plays a critical role in tumor- and growth factor-induced angiogenesis, because antagonists of this integrin potently inhibit angiogenesis and tumor growth. Here we show that the integrin $\alpha 5 \beta 1$ promotes endothelial cell survival during angiogenesis in vivo by suppressing the activity of protein kinase A (PKA). Antagonists of integrin $\alpha 5 \beta 1$ activate PKA, which then leads to the activation of caspase8 and induction of apoptosis. Direct activation of PKA by cAMP or by expression of the PKA catalytic subunit also induces endothelial cell apoptosis, resulting in angiogenesis inhibition in vivo. Our studies indicate that ligation of integrin $\alpha 5 \beta 1$ during angiogenesis suppresses an apoptotic program that is dependent on PKA. These studies also indicate that induction of endothelial cell apoptosis in vivo by genetic or pharmacological activation of PKA may be a useful strategy to inhibit angiogenesis.
\end{abstract}

J. Clin. Invest. 110:933-941 (2002). doi:10.1172/JCI200214268.

\section{Introduction}

New blood vessels develop from preexisting vessels (1) or from circulating endothelial progenitor cells (2) in response to growth factors and/or hypoxic conditions (angiogenesis). Angiogenesis promotes embryonic development, wound healing, and the female reproductive cycle (1), as well as solid tumor cancer, neovascular eye disease, psoriasis, and rheumatoid arthritis (1). While growth factors are required to elicit new blood vessel growth, adhesion to provisional ECM proteins such as fibronectin, vitronectin, and fibrinogen is required for endothelial cell survival, proliferation, and motility during new blood vessel growth (3-8).

The integrin family of ECM receptors mediates not only cellular adhesion to and migration on the ECM proteins found in intercellular spaces and basement membranes, but also cell survival (9-19). Prevention of cell attachment to the ECM induces a form of apoptosis termed anoikis in primary cells and some tumor cells (9-19). However, some integrin antagonists can also suppress cell survival in cells that are still attached to the ECM by other adhesion proteins. For example, antagonists of the integrin

Received for publication September 21, 2001, and accepted in revised form July 30, 2002.

Address correspondence to: Judith A. Varner, University of California, San Diego Cancer Center, 9500 Gilman Drive, La Jolla, California 92093-0912, USA. Phone: (858) 822-0086; Fax: (858) 822-1325; E-mail: jvarner@ucsd.edu.

Conflict of interest: No conflict of interest has been declared. Nonstandard abbreviations used: protein kinase A (PKA); green fluorescent protein (GFP); poly (ADP ribose) polymerase (PARP); human umbilical vein endothelial cell (HUVEC); endothelial basal medium (EBM); defined endothelial growth medium-2 (EGM-2); endothelial growth medium (EGM); PKA catalytic $\left(\mathrm{PKA}_{\text {cat }}\right)$; mutationally inactive PKA (dnPKA); 4',6-diamidine-2phenylindole dihydrochloride (DAPI); chick chorioallantoic membrane (CAM); integrin-mediated death (IMD). $\alpha v \beta 3$ inhibit angiogenesis in vivo, even though endothelial cells remain attached to the ECM through integrins $\alpha 5 \beta 1$ and $\alpha v \beta 5(4,6)$. Similarly, unligated integrin $\alpha 5 \beta 1$ inhibits tumor cell survival and proliferation in vitro and in vivo, even when tumor cells adhere to the ECM through other integrins (20-22). Thus, integrins play key roles in the regulation of cellular survival.

Integrins also regulate vascular development and angiogenesis (4, 6-16). Antagonists of integrin $\alpha v \beta 3$ inhibit tumor angiogenesis and growth by causing endothelial cells in tumors, but not in normal tissues, to die $(4,6)$. Fibronectin and its receptor integrin $\alpha 5 \beta 1$ also regulate angiogenesis (17). Integrin $\alpha 5 \beta 1$ expression is upregulated on human tumor vasculature in many tumors and in healing wounds (17-18). Growth factor and tumor-induced angiogenesis, as well as tumor growth, are inhibited by antagonists of $\alpha 5 \beta 1$ (Ab's, cyclic peptides, and small organic molecules) and by $\mathrm{Ab}$ antagonists of fibronectin, the major ligand for $\alpha 5 \beta 1(17,19)$. Loss of the gene encoding the $\alpha 5$ subunit is also embryonic lethal and is associated with vascular and cardiac defects (23-25). Integrin $\alpha 5 \beta 1$ thus plays a vital role in angiogenesis.

The mechanisms whereby integrin $\alpha 5 \beta 1$ antagonists block angiogenesis are unknown. We show here that antagonists of $\alpha 5 \beta 1$ inhibit endothelial cell survival in vitro and in vivo without affecting cell attachment to the ECM. Perturbing $\alpha 5 \beta 1$ ligation activates cAMP-dependent kinase, protein kinase $\mathrm{A}(\mathrm{PKA})$, which then activates an initiator (caspase-8), but not a stress-mediated (caspase-9) apoptotic pathway. Integrin $\alpha 5 \beta 1$ antagonists thereby suppress cell survival in vitro and during angiogenesis in vivo in a PKA-dependent manner. These studies reveal a novel and important mechanism whereby integrin antagonists induce cell apoptosis and regulate key in vivo processes such as angiogenesis. 


\section{Methods}

General. Anti- $\alpha 5 \beta 1$ and anti- $\alpha 2 \beta 1$ were from Chemicon International (Temecula, California, USA). Anti- $\alpha v \beta 3$, anti-MHC, and N1-green fluorescent protein (N1-GFP) vector were from David Cheresh (The Scripps Research Institute, La Jolla, California, USA). Anti-caspase Ab's were from New England Biolabs Inc. (Beverly, Massachusetts, USA). Anti-poly (ADP ribose) polymerase (PARP) and FITC-annexin V were from PharMingen (San Diego, California, USA). Fibronectin and collagen I were from Collaborative Biomedical Products (Bedford, Massachusetts, USA). Vitronectin was purified from outdated human plasma by denaturing heparin-Sepharose chromatography as described (26). Poly-L-lysine was from Sigma-Aldrich (St. Louis, Missouri, USA). HA1004 was obtained from Biomol Research Laboratories (Plymouth Meeting, Pennsylvania, USA). Caspase inhibitors and activity assays were from Calbiochem-Novabiochem Corp. (La Jolla, California, USA). Ten-day-old chicken embryos were from McIntyre Poultry (Ramona, California, USA), and bFGF was from Genzyme Pharmaceuticals (Cambridge, Massachusetts, USA). Human umbilical vein endothelial cells (HUVECs), endothelial basal medium (EBM), defined endothelial growth medium-2 (EGM-2) (EBM + bFGF, VEGF, and no serum), and endothelial growth medium (EGM) (EBM + bFGF, VEGF, and $2 \%$ serum) were from Clonetics Corp. (San Diego, California, USA). PKA catalytic $\left(P K A_{\text {cat }}\right)$ and mutationally inactive $(d n P K A)$ cDNAs were from Susan Taylor and Renate Pilz (University of California, San Diego, California, USA), respectively. The cDNAs were subcloned into topoTA-pcDNA $3.1 \mathrm{~V} 5 / \mathrm{His}$ according to manufacturer's directions (Invitrogen Corp., Carlsbad, California, USA). HUVECs were cultured in EGM. All statistical analyses were performed using the Student $t$ test.

Annexin V-FITC staining. Culture plates were coated with $10 \mu \mathrm{g} / \mathrm{ml}$ of fibronectin, vitronectin, collagen, or poly-L-lysine at $4^{\circ} \mathrm{C}$ for 16 hours and blocked with denatured BSA. Plates were also coated with $25 \mu \mathrm{g} / \mathrm{ml}$ goat anti-mouse IgG (Southern Biotechnology Associates Inc., Birmingham, Alabama, USA) at $37^{\circ} \mathrm{C}$ for 2 hours, rinsed, blocked with denatured BSA, and then incubated with $10 \mu \mathrm{g} / \mathrm{ml}$ anti- $\alpha 5 \beta 1$ or control $\mathrm{IgG}$ (anti-MHC) at $4^{\circ} \mathrm{C}$ for 16 hours. HUVECs were incubated on plates in the presence or absence of $10 \mu \mathrm{g} / \mathrm{ml}$ anti- $\alpha 5 \beta 1$, anti- $\alpha v \beta 3$, anti- $\alpha 2 \beta 1$, or isotype-matched anti-MHC in defined endothelial growth medium containing bFGF and VEGF for 0-24 hours. In some experiments, HUVECs were incubated with Ab's and $100 \mu \mathrm{M}$ caspase- $3,-8$, or -9 inhibitors or vehicle control $(0.5 \%$ DMSO). Cells were then incubated with FITC-annexin $\mathrm{V}$ for 15 minutes at room temperature in the dark. Washed cells were fixed in $1 \%$ paraformaldehyde, then incubated in 4',6-diamidine-2-phenylindole dihydrochloride (DAPI). The percentage of annexin-positive cells in five microscopic fields was determined at $\times 200$ magnification by fluorescence microscopy.

Apoptosis assays. HUVECs were incubated on ECM or Ab-coated plates for 0-24 hours. DNA fragmentation was detected by agarose gel electrophoresis as described (27). Alternatively, HUVECS on plates were lyzed with RIPA buffer (100 mM Tris, pH 7.2, $150 \mathrm{mM}$ $\mathrm{NaCl}, 1 \%$ deoxycholate; $1 \%$ Triton $\mathrm{X}-100,0.1 \% \mathrm{SDS}$ ) (19) on ice. Lysates were electrophoresed on $8 \%$ SDSPAGE gels and immunoblotted with anti-PARP, -caspase-3, -caspase-9, -cleaved caspase-3, or -cleaved caspase- 9 Ab's. Immunoreactive bands were identified by chemiluminescence and quantified by densitometry. Caspase- 3 and -8 activities were determined in cell lysates according to manufacturer's instructions (Calbiochem-Novabiochem Corp.).

Role of PKA in cell survival. HUVECs were plated for 24 hours on ECM protein-coated plates in the presence of anti- $\alpha v \beta 3$ or anti- $\alpha 5 \beta 1,1 \mu \mathrm{M}$ HA1004 (a PKA inhibitor), and anti- $\alpha v \beta 3$ or anti- $\alpha 5 \beta 1$ with $1 \mu \mathrm{M}$ HA1004. Cells were stained with FITC-annexin V or immunoblotted with anti-cleaved caspase-3 Ab's as described above. HUVECs $\left(5 \times 10^{6}\right)$ were electroporated in $300 \mu \mathrm{l}$ of EGM with $30 \mu \mathrm{g}$ of DNA $(20 \mu \mathrm{g} d n P K A$ or $P K A_{\text {cat }}$ plasmids, $2 \mu \mathrm{g} N 1$-GFP, and $8 \mu \mathrm{g}$ pBluescript) at $300 \mathrm{~V}, 450 \mu \mathrm{F}$, as described previously (28). Expression of transgenes was determined by Western blotting of cell lysates with anti-V5 and anti-GFP Ab's. Transfection efficiency was quantified by determining the percentage of cells that were cotransfected with GFP expression vectors. Eighty percent transfection efficiency was generally achieved using this electroporation method.

Chick chorioallantoic membrane angiogenesis assay. Chick chorioallantoic membrane (CAM) assays were performed as described (17). Saline or bFGF-stimulated CAMs were treated for 24 hours with $20 \mu \mathrm{g}$ anti- $\alpha 5 \beta 1$, anti- $\alpha v \beta 3$, or control Ab's and then injected intravenously with $50 \mu \mathrm{l}$ annexin V-FITC. CAMs were harvested after 2 hours, then analyzed by confocal microscopy. Alternatively, CAMs treated as above were excised, minced on ice, and treated with $0.1 \%$ dispase $/ 0.1 \%$ collagenase for 1 hour at $37^{\circ} \mathrm{C}$. Single-cell suspensions were incubated with FITC-annexin $\mathrm{V}$ and analyzed as described above. The bFGF-stimulated CAMs were also treated with anti- $\alpha 5 \beta 1$ or anti$\alpha v \beta 3$ in the presence or absence of $500 \mu \mathrm{M}$ caspase- $3,-8$ or -9 inhibitors. The bFGF-stimulated CAMs were also transfected with $4 \mu \mathrm{g} N 1-G F P, \operatorname{dn} P K A$, or $P K A_{\text {cat }}$ expression plasmids. Freshly excised CAMs were homogenized in ice-cold RIPA buffer containing protease inhibitors and immunoblotted to detect intact and cleaved caspase3 or -8 . For all treatment groups, $n=10$. CAMs were fixed with $3.7 \%$ paraformaldehyde prior to excision. The average number of blood vessel branch points per treatment group plus or minus SEM is presented. Some excised CAMs were cryopreserved, cut into $5-\mu \mathrm{m}$ sections, immunostained with anti-cleaved caspase-3, anti-cleaved caspase-8, and anti-vFW Ab's (to detect blood vessels). CAMs were also stained by the TUNEL method to detect fragmented DNA.

\section{Results}

Integrin $\alpha 5 \beta 1$ promotes endothelial cell survival in vitro and in vivo. Antagonists of $\alpha 5 \beta 1$ and fibronectin inhibit 
growth factor and tumor-induced angiogenesis $(17,19)$. Because integrins have been shown to promote cell survival, we examined the roles of $\alpha 5 \beta 1$ and fibronectin in endothelial cell survival. Endothelial cells (HUVECs) cultured on poly-L-lysine (which mediates nonspecific cell attachment to the substratum) and fibronectin substrates were analyzed for the binding of annexin $\mathrm{V}$, a $\mathrm{Ca}^{2+}$-dependent phospholipid-binding protein that binds to apoptotic cells with exposed phosphatidyl serine (29). HUVECs attached to fibronectin bind little annexin $\mathrm{V}$, whereas over $85 \%$ of cells on poly-Llysine-coated plates bound annexin V (Figure 1a, $P=0.01$ ). Additionally, lysates from HUVECs cultured in suspension or on poly-L-lysine and fibronectin were immunoblotted for PARP, an enzyme involved in DNA repair that is cleaved by caspase-3 during the early stages of apoptosis to produce $85-\mathrm{kDa}$ and $2-\mathrm{kDa}$ fragments, resulting in loss of normal PARP function $(30,31)$. Cells in suspension or attached to poly-L-lysine displayed significant PARP cleavage, while cells attached to fibronectin showed little PARP cleavage (Figure 1b, $P=0.006$ ). Similarly, attachment to fibronectin, but not poly-L-lysine, protects HUVECs from DNA fragmentation associated with apoptosis (Figure $1 \mathrm{c}, P=0.0001$ ). These studies indicate that fibronectin attachment promotes the survival of proliferating endothelial cells.

To determine if integrin $\alpha 5 \beta 1$ is the fibronectin receptor supporting HUVEC survival in vitro, HUVECs were cultured on surfaces coated with anti- $\alpha 5 \beta 1$ or control Ab's. Immobilized anti-integrin Ab's cluster integrins, thereby acting as agonists $(6,32)$. Cells attached to surfaces coated with Ab's directed against $\alpha 5 \beta 1$ bound little annexin (Figure $1 \mathrm{~d}, P=0.001$ ) and thus remained viable. In contrast, more than $75 \%$ of cells attached to control Ab's were annexin positive. Cells attached to $\alpha 5 \beta 1 \mathrm{Ab}$-coated surfaces also showed significantly less PARP cleavage (Figure 1e, $P=0.007$ ) and DNA fragmentation (Figure 1f, $P=0.003$ ) than cells attached to control Ab's. These results indicate that $\alpha 5 \beta 1$ promotes endothelial cell survival.

Antagonists of integrin $\alpha 5 \beta 1$ block angiogenesis in vivo but have no effect on unstimulated blood vessels (17). To determine if these antagonists induce endothelial cell apoptosis during angiogenesis in vivo, CAMs were stimulated with $\mathrm{bFGF}$, treated with saline, function-blocking anti- $\alpha 5 \beta 1 \mathrm{Ab}$ 's, and isotype-matched control Ab's, and then analyzed for markers of apoptosis. Intravenous injection of fluorescently labeled annexin $V$ demonstrated that $\alpha 5 \beta 1$ antagonists induce endothelial cell apoptosis in vivo. Anti- $\alpha 5 \beta 1 \mathrm{Ab}$ 's, but not saline or control Ab's, induced annexin $\mathrm{V}$ staining of blood vessels in living CAMs, indicating that $\alpha 5 \beta 1$ regulates survival in vivo of proliferating endothelial cells (Figure 2a). In fact, vessels in anti- $\alpha 5 \beta 1$-treated CAMs bound eight times more annexin $V$ than controltreated CAMs (Figure $2 \mathrm{~b}, P=0.015$ ). These anti- $\alpha 5 \beta 1$
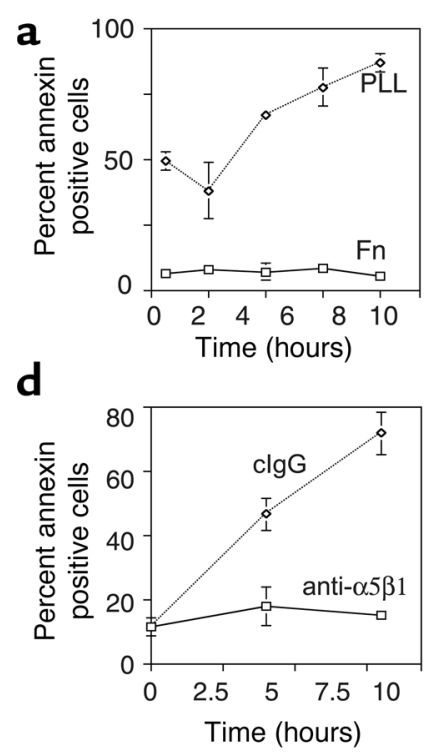

b

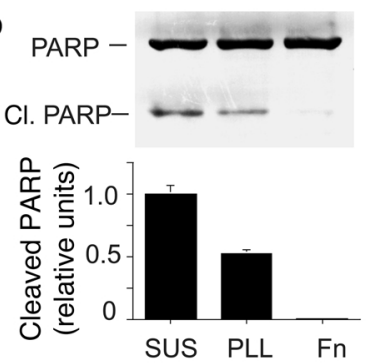

e

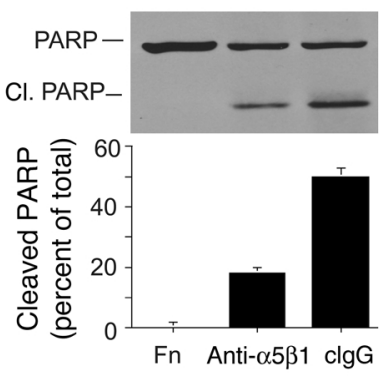

C
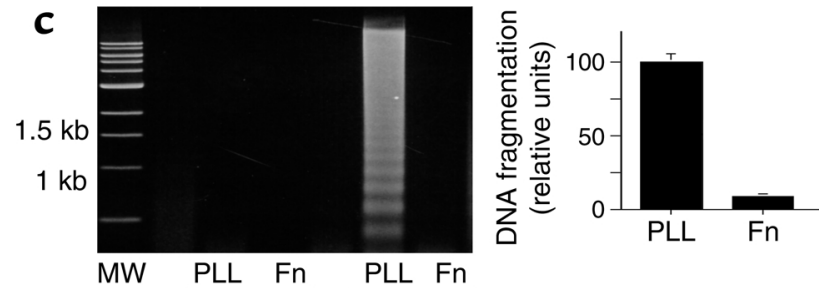

\section{Figure 1}

Fibronectin and integrin $\alpha 5 \beta 1$ support endothelial cell survival. (a-c) HUVECs were maintained in suspension (SUS) or on fibronectin-coated (Fn) or poly-L-lysine-coated (PLL) plates. (a) The percentage of annexin V-positive cells on poly-L-lysine- or fibronectin-coated dishes was determined at regular intervals from 0 to 8 hours. (b) Cell lysates prepared after 4 hours of attachment were immunoblotted to detect intact (116 kDa) and cleaved PARP ( $85 \mathrm{kDa})$. The ratio of intact to cleaved PARP was determined by densitometry. Cl., cleaved. (c) Soluble DNA extracted from cells attached to poly-L-lysine or fibronectin was electrophoresed on 1.6\% agarose gels. Relative DNA cleavage was determined by densitometry. (d-f) HUVECs were plated on fibronectin, anti- $\alpha 5 \beta 1$, or control Ab-coated plates. (d) The percentage of annexin V-positive cells was determined from 0 to 8 hours. (e) Cell lysates were immunoblotted to detect intact and cleaved PARP. (f) DNA fragmentation was evaluated as in c. clgG, control immunoglobulin. 

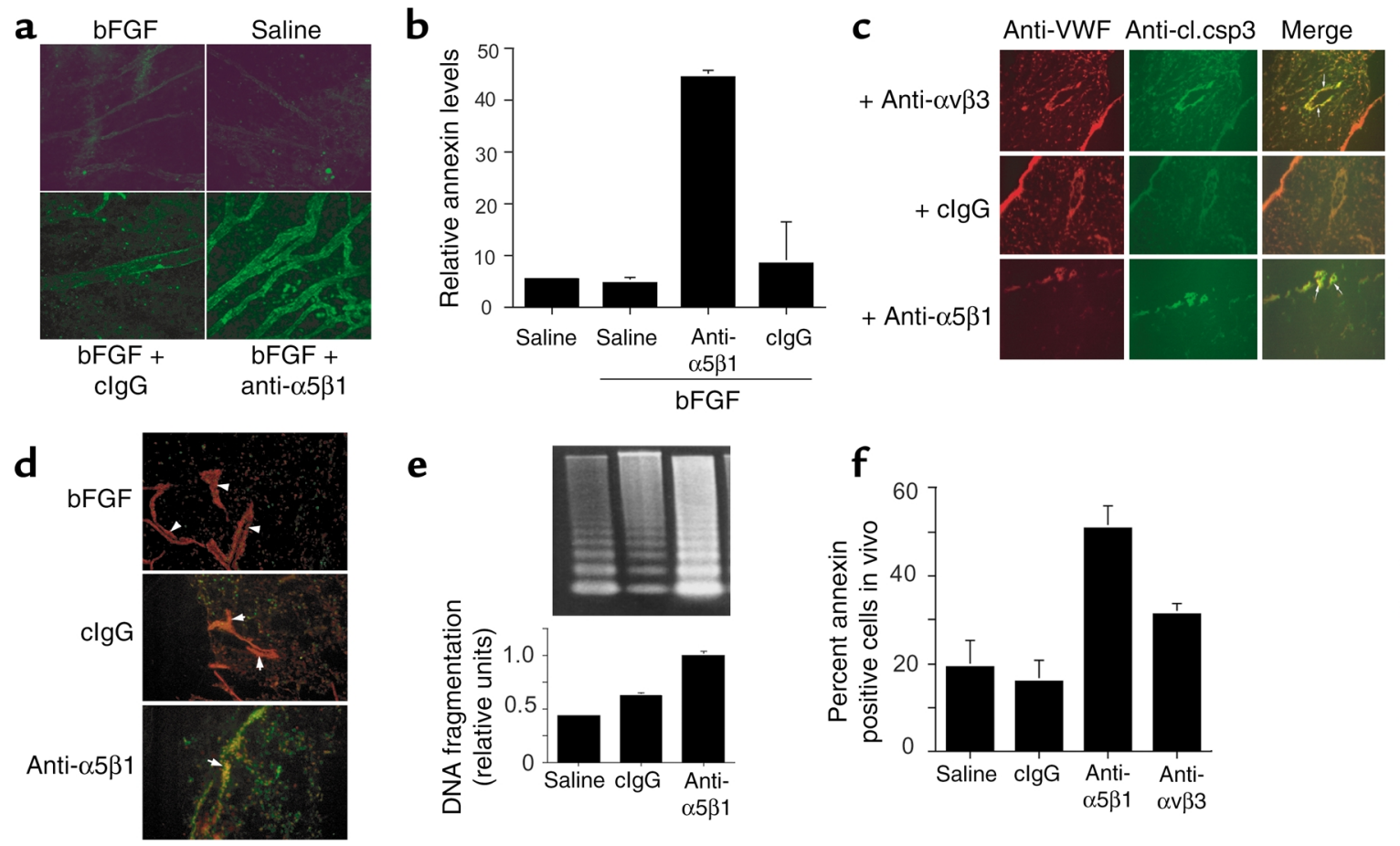

\section{Figure 2}

Integrin $\alpha 5 \beta 1$ supports endothelial cell survival during angiogenesis in vivo. (a) CAMs were stimulated with bFGF or saline and then treated for 24 hours with saline, anti- $\alpha 5 \beta 1$, and control Ab's. CAMs were then injected with $50 \mu$ I FITC-annexin V, harvested 2 hours later, and analyzed directly by confocal microscopy. (b) Green pixels (annexin $\vee$ positive) present per optical section were quantified. (c) CAMs treated as in a were cryosectioned and immunostained with anti-cleaved caspase-3 (anti-cl.csp3) (green) and anti-vWF (red). Cleaved caspase3-positive blood vessels are yellow (arrows). (d) CAMs treated as in a were cryosectioned and immunostained with anti-vWF (red) and for DNA fragmentation (TUNEL staining, green). Arrows indicate blood vessels. Yellow structures are apoptotic blood vessels. (e) Soluble DNA isolated from CAMs treated as in a was electrophoresed on 1.6\% agarose gels. Molecular-weight markers are 1-kb DNA ladders. Relative DNA cleavage was determined by densitometry. (f) Individual cells isolated from CAMs treated as in a were stained with FITC-annexin V.

Ab's were shown previously to react with $\alpha 5 \beta 1$ on chicken endothelial cells and to block its function in vitro and in vivo (17). Because $\alpha 5 \beta 1$ is only expressed at significant levels on proliferating endothelial cells (17), $\alpha 5 \beta 1$ function-blocking Ab's target proliferating endothelial cells. Because peptide and small molecule antagonists of $\alpha 5 \beta 1$ also inhibit angiogenesis (17) and induce apoptosis (not shown), Ab-mediated complement activation is not likely to play a significant role in this apoptosis induction. Thus, these results suggest that integrin $\alpha 5 \beta 1$ antagonists induce endothelial cell apoptosis in vivo.

To determine further whether $\alpha 5 \beta 1$ antagonists cause apoptosis in vivo, sections of CAMs were analyzed for the expression of cleaved caspase- 3 in blood vessels (Figure $2 c, P=0.01$ ). Cleavage of caspase- 3 into 17 - and $12-\mathrm{kDa}$ fragments is an indication of caspase- 3 activation; the amount of cleaved caspase- 3 is a quantitative index of apoptosis induction. Ab's directed against mammalian caspases cross-react with avian caspases, because avian caspases exhibit $65 \%$ overall sequence identity and $100 \%$ activation domain sequence identity with mammalian caspases (33). Treatment with either $\alpha 5 \beta 1$ or $\alpha v \beta 3$ antagonists induces caspase- 3 cleavage (green) in blood vessels (red) in growth factor-stimulated CAMs. Antagonists of $\alpha 5 \beta 1$ but not control Ab's also induce DNA fragmentation in bFGF-stimulated endothelial cells, as evaluated by TUNEL staining (Figure $2 \mathrm{~d}$ ) and by analysis of DNA fragmentation of agarose gels (Figure 2e, $P=0.003$ ). Furthermore, isolated cells from CAMs treated with $\alpha 5 \beta 1$ and $\alpha v \beta 3$ function-blocking Ab's bound significantly more FITCannexin $V$ than in cells from control CAMs (Figure $2 f$, $P=0.0002$ and 0.003 , respectively). These studies definitively show that $\alpha 5 \beta 1$ antagonists induce endothelial cell apoptosis during angiogenesis.

Unligated integrin $\alpha 5 \beta 1$ induces apoptosis in adherent endothelial cells. To determine how $\alpha 5 \beta 1$ antagonists interfere with cell survival during angiogenesis, proliferating HUVECs in complete culture medium were plated on poly-L-lysine-, fibronectin-, vitronectin-, or collagen-coated culture plates in the presence of $\mathrm{Ab}$ antagonists of integrins $\alpha 5 \beta 1, \alpha v \beta 3$, or $\alpha 2 \beta 1$ for 24 hours. Cells were then stained with FITC-annexin V. While fibronectin and vitronectin promote the survival of proliferating endothelial cells (Figure 3, a and c), collagen does not (ref. 34; data not shown). Integrin antagonists that block cell attachment to the substratum (Figure 3, b and d) induce apoptosis, or anoikis, in endothelial cells (Figure 3, a and c). For example, antagonists of $\alpha 5 \beta 1$ induce apoptosis on fibronectin (Figure 3a, $P=0.001$ ), while antagonists of $\alpha v \beta 3$ induce apop- 

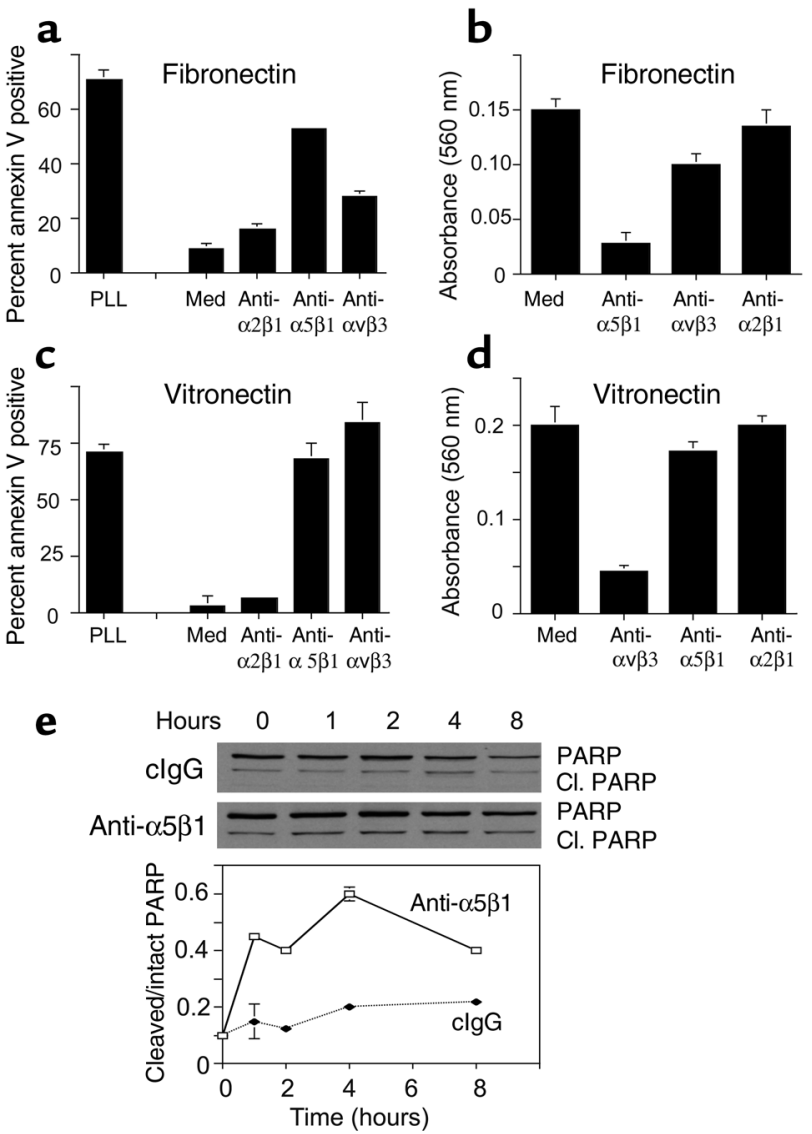

Figure 3

Unligated integrin $\alpha 5 \beta 1$ regulates endothelial cell survival. HUVECs were plated on poly-L-lysine-coated, fibronectin-coated ( $\mathbf{a}$ and $\mathbf{b}$ ), or vitronectin-coated ( $\mathbf{c}$ and $\mathbf{d}$ ) culture plates in culture medium (med) or culture medium containing anti- $\alpha 5 \beta 1$, anti- $\alpha v \beta 3$, anti- $\alpha 2 \beta 1 \mathrm{Ab}$ 's. After 1 hour, cell attachment was determined ( $\mathbf{b}$ and $\mathbf{d}$ ). After 24 hours, the percentage of FITC-annexin V-positive ( $\mathbf{a}$ and $\mathbf{c}$ ) cells was determined. (e) HUVECs plated on vitronectin-coated plates in the presence of function-blocking anti- $\alpha 5 \beta 1$ or control Ab's were collected at regular intervals from 0 to 8 hours and PARP cleavage assessed by Western blotting. Relative PARP cleavage levels were determined by densitometry.

tosis on vitronectin (Figure $3 c, P=0.02$ ) by blocking cell attachment to the substratum.

In vivo, however, anoikis induced by loss of attachment to the ECM is unlikely to occur because endothelial cells are attached to multiple matrix proteins through multiple integrins at any one time. Therefore, we also examined the ability of integrin antagonists to induce apoptosis in cells that remain attached through other integrins. In fact, $\alpha 5 \beta 1$ antagonists induce apoptosis of cells on vitronectin (Figure $3 c, P=0.05$ ) without affecting their attachment to vitronectin (Figure $3 d$ ). Anti- $\alpha 5 \beta 1$, but not control Ab's, also induce PARP cleavage in HUVECs plated on vitronectin substrates (Figure $3 e, P=0.003$ ). Thus, unligated $\alpha 5 \beta 1$ inhibits endothelial cell survival on provisional matrix proteins such as fibronectin and vitronectin. These studies indicate that integrin $\alpha 5 \beta 1$ provides critical survival signals to proliferating endothelial cells such as those partici- pating in angiogenesis. These studies indicate that integrin $\alpha 5 \beta 1$ can directly and indirectly regulate survival of proliferating endothelial cells.

Unligated integrin $\alpha 5 \beta 1$ activates a caspase-8-mediated apoptosis pathway in vitro and in vivo. To determine the nature of the cell death pathway induced by $\alpha 5 \beta 1$ antagonists, endothelial cells were plated on ECM protein-coated culture plates in the presence of integrin antagonists and caspase inhibitors or vehicle control $(0.33 \% \mathrm{DMSO})$. Cell death induced by anti- $\alpha 5 \beta 1 \mathrm{Ab}$ 's was blocked by caspase- 3 inhibitors, whether the cells were attached to fibronectin $(P=0.0005$, Figure $4 a)$ or vitronectin $(P=0.02$, Figure $4 \mathrm{~b})$. Cell attachment to poly-L-lysine rapidly activated caspases- 3 and -8 while attachment to vitronectin did not (Figure 4, $\mathrm{c}$ and d). Anti- $\alpha 5 \beta 1 \mathrm{Ab}$ 's but not control Ab's also activated cas-
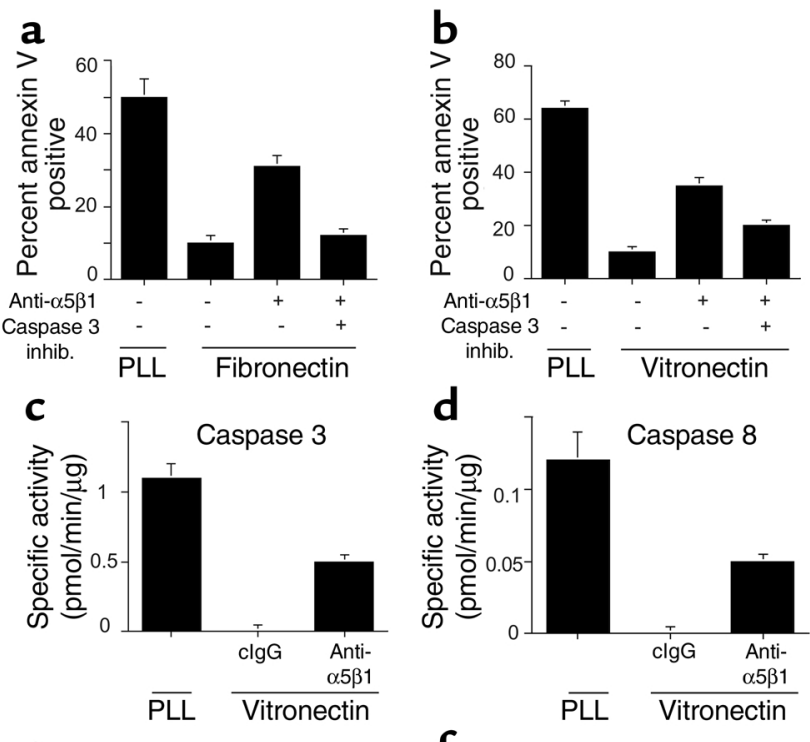

\section{e}

Cl. caspase 3
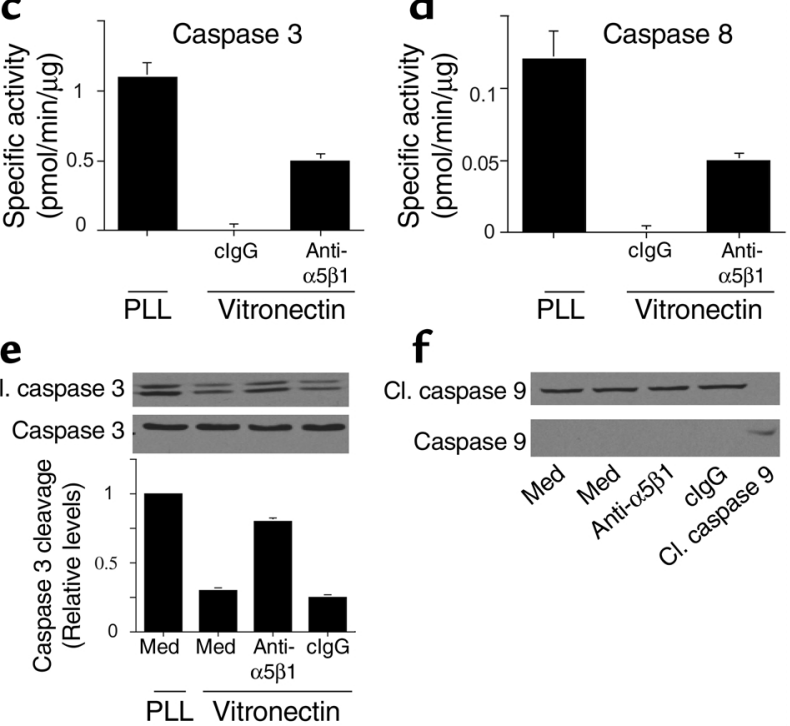

f

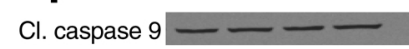

Caspase 9

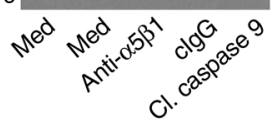

Figure 4

Unligated integrin $\alpha 5 \beta 1$ induces caspase- 3 and -8 , but not -9 , activation. HUVECs were plated on (a) fibronectin-, (b) vitronectin-, or $(\mathbf{a}$ and $\mathbf{b})$ poly-L-lysine-coated culture plates in the presence of anti$\alpha 5 \beta 1 \mathrm{Ab}$ 's and $50 \mu \mathrm{M}$ z-DEVD-fmk (caspase- 3 ) or z-IETD-fmk (caspase-8) inhibitors or vehicle control (0.33\% DMSO) for 24 hours. The percentage of annexin $\mathrm{V}$-positive cells was then determined. inhib, inhibitor. (c and d) Caspase- 3 and -8 activities were determined in HUVECs plated on vitronectin-coated or poly-L-lysine-coated plates in the presence of culture medium, anti- $\alpha 5 \beta 1$, or control Ab's. (e) Cell lysates were immunoblotted with anti-caspase- 3 and anti-cleaved caspase-3 Ab's. Relative caspase-3 cleavage was determined by densitometry. (f) Cell lysates were immunoblotted with anti-caspase- 9 and anti-cleaved caspase- 9 Ab's. 


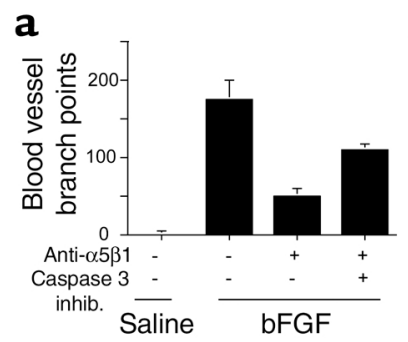

b

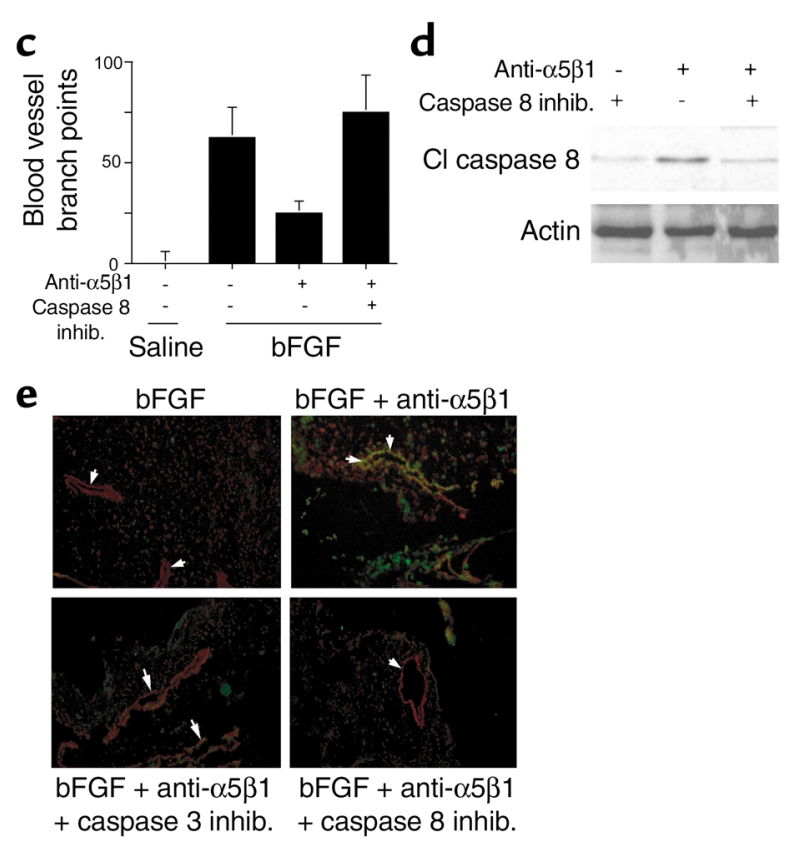

Figure 5

Integrin antagonists induce caspase-3- and -8-dependent apoptosis in vivo. (a-d) CAMs stimulated with saline or bFGF were treated with $2.5 \%$ DMSO (vehicle control), anti- $\alpha 5 \beta 1$, or anti- $\alpha 5 \beta 1$ with $500 \mu \mathrm{M}$ caspase- 3 ( $\mathbf{a}$ and $\mathbf{b}$ ) or caspase- 8 ( $\mathbf{c}$ and $\mathbf{d}$ ) inhibitors. ( $\mathbf{a}$ and $\mathbf{c}$ ) Blood vessel branch points were quantified after 48 hours. (b and $\mathbf{d}$ ) Caspase- 3 and -8 cleavage was evaluated by Western blotting with (b) anti-cleaved caspase-3 or (d) anti-cleaved caspase-8 Ab's and anti-actin Ab's. (e) Cryosections of CAMs treated as in a-d were immunostained for VWF expression (red) and to detect DNA fragmentation (TUNEL staining, green). Arrows indicate blood vessels. Apoptotic vessels appear yellow.

pase- 3 and $-8(P=0.0001$ and $P=0.002$, respectively $)$ in cells attached to vitronectin (Figure 4, $\mathrm{c}$ and $\mathrm{d}$ ) and fibronectin (not shown). Caspase-3 cleavage was also readily detected in cells treated with anti- $\alpha 5 \beta 1$ but not control Ab's $(P=0.01$, Figure $4 \mathrm{e})$. In contrast, caspase- 9 cleavage was not detected in cells treated with $\alpha 5 \beta 1$ antagonists (Figure 4f). These studies show that integrin $\alpha 5 \beta 1$ antagonists induce a proapoptotic pathway in proliferating endothelial cells that results from activation of initiator caspases (8) rather than stress caspase (9) pathways. These results also indicate that blocking $\alpha 5 \beta 1$ ligation induces caspase-8- and -3-mediated death even when cells are still attached to provisional matrix ligands through other integrins.

Our studies show that integrin-mediated survival depends on suppression of caspase- 3 and -8 activity in vitro. As $\alpha 5 \beta 1$ antagonists block angiogenesis in vivo, these antagonists may induce caspase- 3 and -8 activation in vivo. CAMs stimulated with $\mathrm{bFGF}$ were treated with saline, vehicle control (DMSO), caspase-3 or -8 inhibitors, and anti-integrin Ab's in the presence or absence of caspase inhibitors. Angiogenesis was inhibited by anti- $\alpha 5 \beta 1 \mathrm{Ab}$ 's (Figure 5 , $\mathrm{a}-\mathrm{d}$ ); this inhibition was partially reversed by cell-permeable caspase- 3 inhibitors $(P=0.04$, Figure 5a) and fully reversed by caspase- 8 inhibitors $(P=0.05$, Figure $5 c)$. Caspase- 3 inhibitors partially blocked caspase- 3 activity in vivo (Figure $5 b$ ), while caspase-8 inhibitors completely blocked its activity in vivo (Figure 5d). Furthermore, caspase-3 and -8 inhibitors prevented in vivo endothelial cell DNA fragmentation induced by $\alpha 5 \beta 1$ inhibition (Figure 5e). Caspase-9 inhibitors had little effect on angiogenesis (not shown). Caspase inhibitors alone had no effect on angiogenesis or on unstimulated CAMs. These results indicate that $\alpha 5 \beta 1$ antagonists activate caspases- 8 and -3 in vivo, thereby inhibiting angiogenesis.

Unligated integrins induce PKA-dependent apoptosis. Integrin ligation activates signaling pathways that promote cell migration, proliferation, and survival. Typically, integrin-mediated signaling is characterized by increases in tyrosine phosphorylation of signaling intermediates such as focal adhesion kinase, src, and ERK family members. However, integrin ligation also suppresses the activation of at least one kinase, PKA (19). Importantly, we found that integrin ligation suppresses PKA activation, while antagonists of integrins activate this enzyme (Figure 6a). Notably, antagonists of integrin $\alpha 5 \beta 1$ activate endothelial cell PKA whether endothelial cells are plated on fibronectin or on vitronectin (Figure 6a). Therefore, we investigated the contribution of PKA to integrinmediated cell death. We found that a pharmacological inhibitor of PKA, HA1004, substantially suppressed the apoptosis induced by integrin antagonists anti- $\alpha v \beta 3$ $(P=0.05)$ or anti- $\alpha 5 \beta 1(P=0.05)$ in cells attached to vitronectin, as detected by annexin $V$ binding to intact cells (Figure 6b). PKA inhibitors also blocked caspase- 3 cleavage induced by anti- $\alpha 5 \beta 1(P=0.002$, Figure $6 c)$. Expression of dnPKA, but not a control transgene (GFP), also prevented integrin antagonist-induced cell death $(P=0.004$; Figure 7 , a and $b)$. More than $80 \%$ of cells expressed the transgene, which was also detected by Western blotting (Figure 7d). These studies indicate that both direct (anoikis) and indirect integrin antagonist-mediated cell death is PKA dependent.

PKA activation induces endothelial cell apoptosis in vitro and in vivo. Our studies show that inhibition of PKA suppresses integrin antagonist-induced cell death. To determine whether activation of PKA directly induces endothelial cell death, endothelial cells were treated with dibutyryl cAMP or were transiently transfected with the active, catalytic subunit of PKA. Both cAMP and expression of the catalytic subunit of PKA significantly induce apoptosis $(P=0.009$ and $P=0.003$, respectively) in endothelial cells in vitro (Figure 7c). Thus, PKA directly induces apoptosis in endothelial cells. 


\section{$\mathbf{a}$}

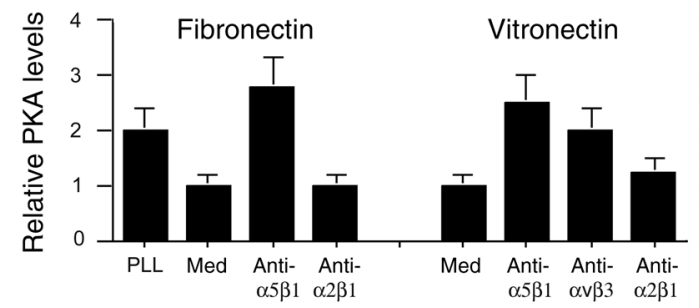

b

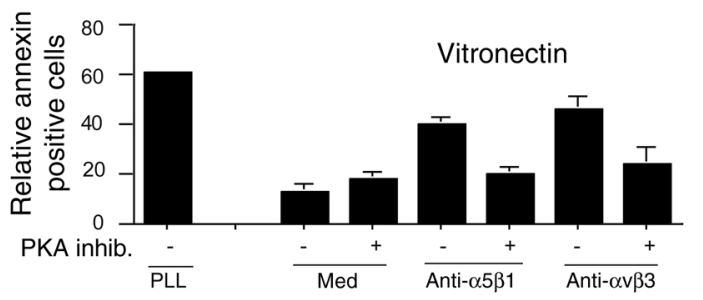

C

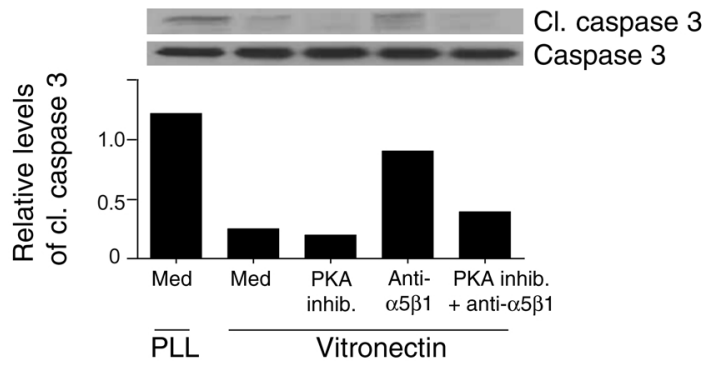

Figure 6

Unligated $\alpha 5 \beta 1$-mediated death is PKA dependent. (a) PKA activity was measured in HUVECs attached to poly-L-lysine, fibronectin, or vitronectin in the presence or absence of integrin antagonists. (b) HUVECs were plated on vitronectin-coated or poly-L-lysine-coated culture plates in the presence or absence of anti- $\alpha 5 \beta 1$ or anti- $\alpha v \beta 3$

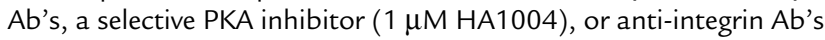
in combination with $1 \mu \mathrm{M} \mathrm{HA} 1004$. After 24 hours, the percentage of FITC-annexin-positive cells was determined. (c) Cell lysates from $\mathbf{b}$ were immunoblotted with anti-caspase- 3 and anti-cleaved caspase3 Ab's. Relative caspase- 3 cleavage was determined by densitometry.

To determine whether PKA plays a role in the negative regulation of angiogenesis in vivo, bFGF-stimulated CAMs were transfected with expression plasmids encoding GFP and dnPKA and treated with $\alpha 5 \beta 1 \mathrm{Ab}$ 's. While $\alpha 5 \beta 1$ antagonists block angiogenesis (Figure 8a, $P=0.004$ ), dnPKA reverses this inhibition (Figure 8a, $P=0.02)$. Expression of dnPKA also prevents $\alpha 5 \beta 1$ antagonist-induced DNA fragmentation in vivo as detected by TUNEL staining (Figure $8 \mathrm{~b}$ ) and inhibits activation of caspase-3 and -8 in vivo (Figure 8c). Since integrin antagonists induce PKA-dependent apoptosis in vivo, direct activation of PKA may also inhibit angiogenesis. Expression of the PKA catalytic subunit during angiogenesis in vivo completely suppresses angiogenesis $(P=0.0005)$, as does exposure to cAMP $(P=0.001$, Figure $8 \mathrm{~d})$. VEGFstimulated angiogenesis is also inhibited by activation of PKA (data not shown). This inhibition results from apoptosis induction, because it is accompanied by DNA fragmentation detected by TUNEL staining (Figure 8e) and caspase-3 cleavage in vivo (Figure 8f). The expression of transgenes could be demonstrated in endothelial cells in vivo by immunohistochemical staining for the presence of His-tagged proteins (Figure 8g). Thus, activation of PKA by integrin antagonists, by cAMP, or by expression of the catalytic subunit of PKA induces endothelial cell apoptosis and inhibits angiogenesis.

These studies demonstrate that the unligated integrin $\alpha 5 \beta 1$ induces endothelial cell apoptosis in vivo in a PKA-dependent manner, thereby inhibiting angiogenesis. These studies demonstrate that activation of PKA by unligated integrins is an essential step in the induction of apoptosis. Importantly, our studies demonstrate that direct activation of PKA in vivo induces endothelial cell apoptosis and inhibition of angiogenesis.

\section{Discussion}

During normal embryonic development and during adult tissue repair processes, unwanted cells are elimi-
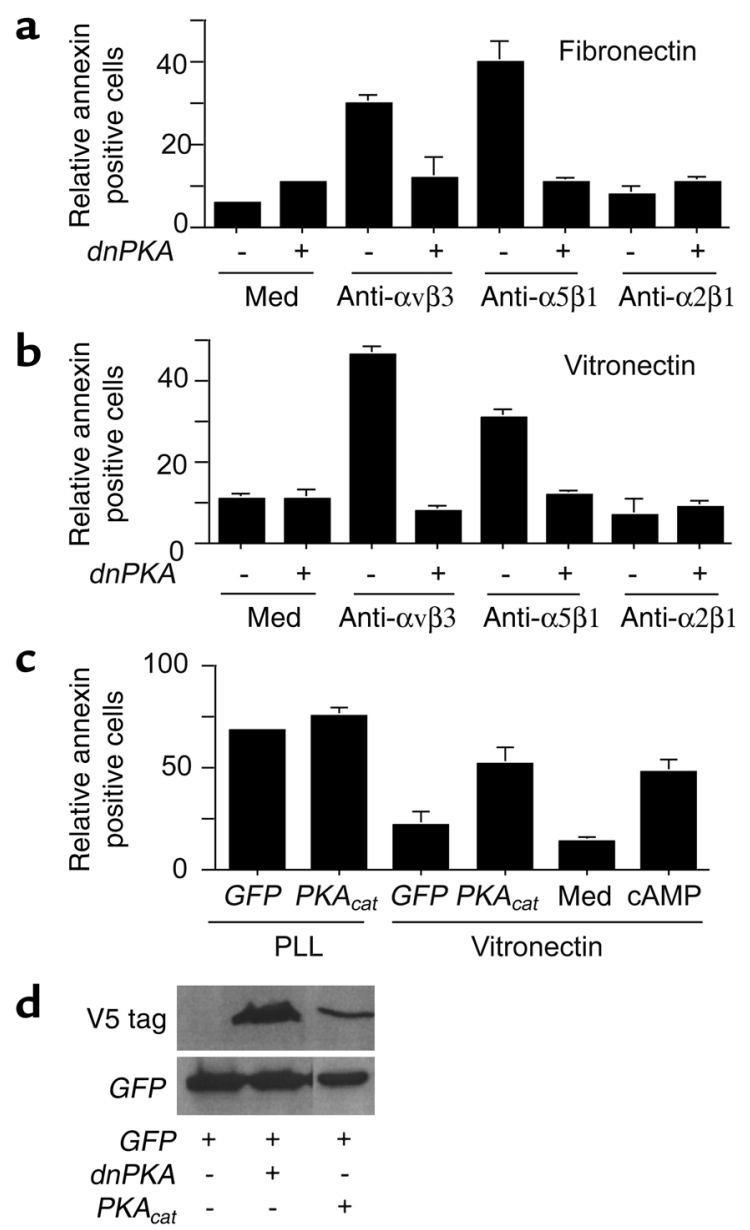

Figure 7

PKA negatively regulates cell survival. ( $\mathbf{a}$ and $\mathbf{b}$ ) HUVECs transfected with GFP (-) or a $\operatorname{dnPKA}(+)$ were plated on (a) fibronectin-coated, (b) vitronectin-coated, or poly-L-lysine-coated plates in the absence or presence of anti- $\alpha 5 \beta 1$, anti- $\alpha v \beta 3$, or anti- $\alpha 2 \beta 1$. After 24 hours, the percentage of annexin $\mathrm{V}$-positive cells was determined. (c) HUVECs treated with culture medium or dibutyryl cAMP $(250 \mu \mathrm{M})$ and HUVECs transfected with GFP or the catalytic subunit of PKA $\left(P K A_{c a t}\right)$ were plated on vitronectin-, or poly-L-lysine-coated plates. After 24 hours, the percentage of annexin $V$-positive cells was determined. (d) Expression of transgenes was detected by Western blotting cell lysates with anti-GFP or anti-V5. 

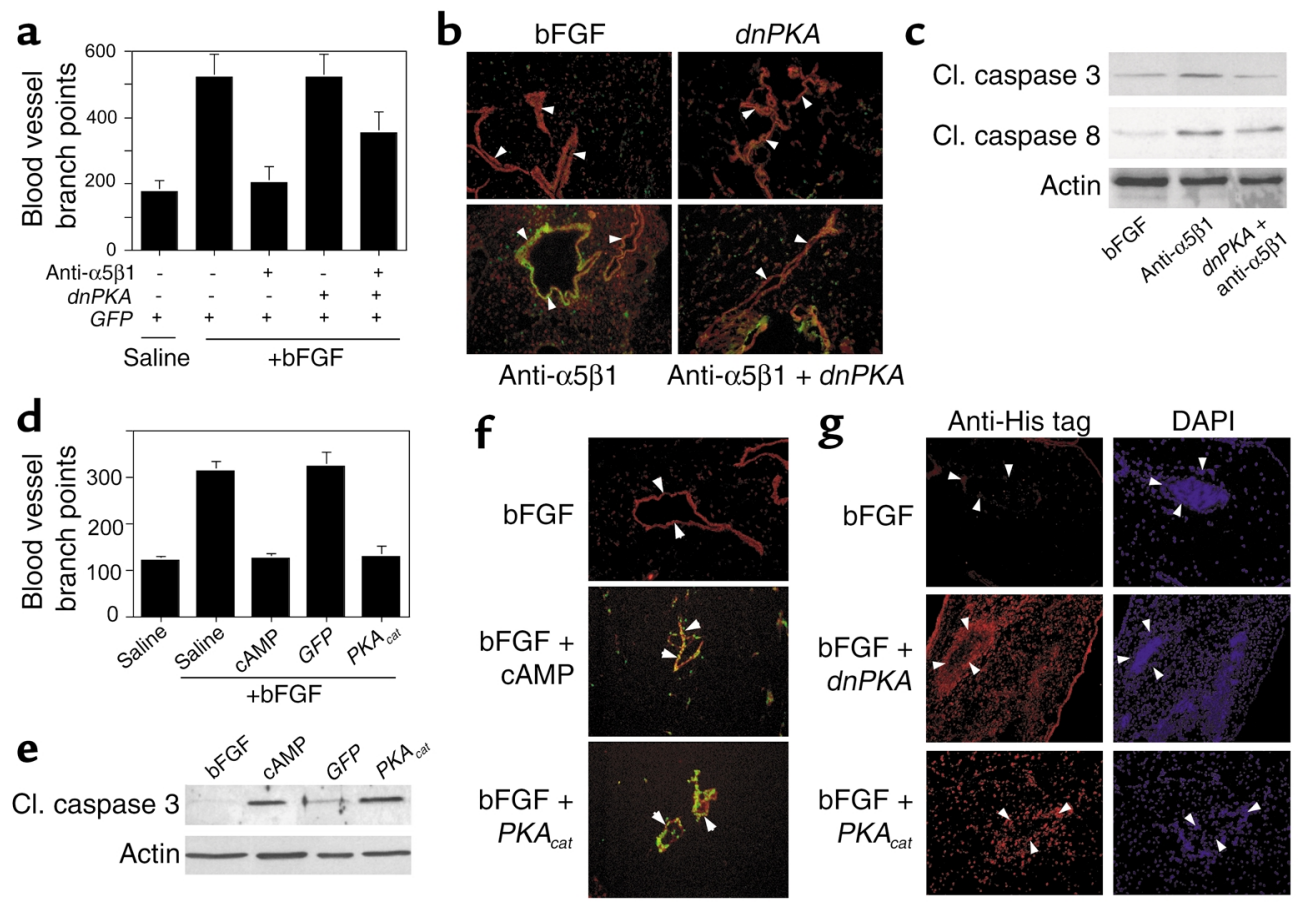

\section{Figure 8}

PKA inhibits angiogenesis by inducing apoptosis. (a) CAMs stimulated with bFGF were transfected 24 hours later by placing $4 \mu \mathrm{g}$ $p c D N A / V 5 / H i s ~ d n P K A$ or N1-GFP expression plasmid on CAMs. CAMs were treated on the same day with saline or anti- $\alpha 5 \beta 1 \mathrm{Ab}$ 's and were harvested 48 hours later. Blood vessel branch points were quantified. (b) Cryosections of CAMs from a were immunostained with anti-vWF (red) and were stained to detect fragmented DNA by the TUNEL method (green). Arrows indicate blood vessels. Apoptotic blood vessels appear yellow. (c) Western blots of lysates prepared from CAMs treated as in a were immunoblotted with anti-cleaved caspase-3 and anti-cleaved caspase -8 , as well as anti-actin as a loading control. (d) CAMs stimulated with bFGF were treated with saline or $250 \mu \mathrm{M}$ cAMP or were transfected by placing $4 \mu \mathrm{g} p c D N A / V 5 / H i s$ PKA cat or N1-GFP expression plasmid on stimulated CAMs. Blood vessel branch points were quantified 48 hours later. (e) Cryosections of CAMs from $\mathbf{d}$ were immunostained with anti-vWF (red) and were stained to detect fragmented DNA by the TUNEL method (green). Arrows indicate blood vessels. Apoptotic blood vessels appear yellow. (f) Detergent lysates prepared from freshly excised CAMs from $\mathbf{d}$ were immunoblotted for expression of cleaved caspase- 3 and actin as a loading control. (g) Cryosections of CAMs from a and $\mathbf{d}$ were immunostained with anti-pentaHis (red) to detect expression of His-tagged transgenes in the transfected CAM tissue. Sections were counterstained with DAPI. Arrows indicate blood vessels.

nated by apoptosis (35). Cell death is also induced by environmental stress, activation of death receptors, and loss of contact with the ECM (35). Apoptosis is initiated by activation of caspases, cysteine proteins that cleave target proteins after aspartic acid residues (36). Initiator and stress caspases are activated by extracellular stimuli and, in turn, activate effector caspases. Effector caspases cleave important cellular proteins such as focal adhesion kinase and p21-activated kinase, which regulate cell shape as well as enzymes that are important for DNA repair and nuclear integrity (36). Integrin-mediated cell attachment promotes cell survival (37), while interruption of cell attachment induces anoikis or detachment-induced apoptosis $(38,39)$. Our studies indicate that key unligated integrins can also induce cell death when cells are still attached to the ECM. We show here that unligated integrin $\alpha 5 \beta 1$ inhibits endothelial cell survival and angiogenesis in ECM-adherent cells by activating PKA and, subsequently, caspase-8. In proliferating endothelial cells, $\alpha 5 \beta 1$ is ligated by endothelial cell-secreted fibronectin $(19,40)$. Antagonists of either $\alpha 5 \beta 1$ or fibronectin inhibit $\alpha 5 \beta 1$ function, thereby activating PKA and inducing cell death, even when cells remain attached to the ECM through integrin $\alpha v \beta 3$ or other integrins. Recent studies suggest that unligated integrin $\alpha v \beta 3$ actively induces apoptosis by a pathway termed integrin-mediated death (IMD). IMD may be initiated by the interaction of unligated integrin $\beta$ subunit cytoplasmic tails with caspase-8 (41). Our studies indicate that unligated $\alpha 5 \beta 1$ also induces IMD and that IMD is dependent on the activity of PKA.

Here we show that direct activation of PKA with cAMP or by overexpression of the PKA catalytic subunit induces apoptosis in proliferating endothelial cells and inhibits angiogenesis. PKA activation has been implicated previously in the regulation of apoptosis in some, but not all, transformed cells (42). In some cells, PKA phosphorylates and inhibits the tyrosine kinase Raf, thereby blocking the MAP kinase pathway and inducing apoptosis (43). Alternatively, PKA may negatively regulate cell survival by hyperphosphorylating Bcl-2 and suppressing $\mathrm{Bcl}-2$ binding to Bax, thus lowering the $\mathrm{Bcl}-2 / \mathrm{Bax}$ ratio (44). Since integrin ligation activates $\mathrm{Bcl}-2$ (45), integrin antagonism may inhibit Bcl-2 in a PKA-dependent manner. PKA activation also inhibits Akt, a prosurvival serine/threonine kinase that phosphorylates and inacti- 
vates $\mathrm{BAD}$, a member of the proapoptotic $\mathrm{Bcl}-2$ family (46). Phosphorylation of BAD inhibits its association with and inactivation of the prosurvival proteins, $\mathrm{Bcl}-2$ and Bcl-x (46). Alternatively, since PKA and caspase- 8 are rapidly activated upon inhibition of integrin ligation (19), PKA may play an early role in endothelial cell death by activating caspase- 8 . Thus, PKA may negatively regulate one or more cell survival signaling pathways.

Unligated integrins may play important roles in suppressing cell survival during tissue remodeling, such as occurs during development, angiogenesis, and wound healing. In these circumstances, cell viability may be promoted when cells remain in the proper environment. Primary cells that inappropriately wander into other microenvironments lacking the correct ECM ligands would be induced to undergo apoptosis. Tumor cells that are more resistant to apoptotic stimuli than normal cells may escape this IMD pathway and wander to distant environments, causing metastases. The studies presented here indicate that in primary cells, unligated integrins induce apoptosis by a PKA-dependent and caspase-8-dependent pathway.

\section{Acknowledgments}

This work was supported by grants CA-71619 and CA83133 from the NIH to J.A. Varner. J.A. Varner is a member of the Comprehensive Cancer Center of the University of California, San Diego.

1. Varner, J.A. 1997. The role of vascular cell integrin $\alpha v \beta 3$ and $\alpha v \beta 5$ in angiogenesis. EXS. 79:361-390.

2. Asahara, T., et al. 1999. VEGF contributes to postnatal neovascularization by mobilizing bone marrow derived endothelial progenitor cells. EMBOJ. 18:3964-3972.

3. Meredith, J.E., Jr., Fazeli, B., and Schwartz, M.A. 1993. The ECM as a cell survival factor. Mol. Biol. Cell. 4:953-961.

4. Brooks, P.C., et al. 1994. Integrin $\alpha v \beta 3$ antagonists promote tumor regression by inducing apoptosis of angiogenic blood vessels. Cell. 79:1157-1164.

5. Boudreau, N., Sympson, C.J., Werb, Z., and Bissell, M. 1995. Suppression of ICE and apoptosis in mammary epithelial cells by ECM. Science. 267:891-893.

6. Stromblad, S., Becker,J.C., Yebra, M., Brooks, P.C., and Cheresh, D.A. 1996. Suppression of $\mathrm{p} 53$ activity and $\mathrm{P} 21 \mathrm{WAF} 1 / \mathrm{CIP} 1$ expression by vascular cell integrin $\alpha v \beta 3$ during angiogenesis. J. Clin. Invest. 98:426-433.

7. Clark, R.A.F., Tonneson, M.G., Gailit, J., and Cheresh, D.A. 1996. Transient functional expression of $\alpha \mathrm{v} \beta 3$ on vascular cells during wound repair. Am. J. Path. 148:1407-1421.

8. Friedlander, M., et al. 1995. Definition of two angiogenic pathways by distinct alpha $v$ integrins. Science. 270:1500-1502.

9. Arap, W., Pasqualini, R., and Ruoslahti, E. 1997. Cancer treatment by targeted drug delivery to tumor vasculature in a mouse model. Science. 279:377-380.

10. Brooks, P.C., Clark, R.A., and Cheresh, D.A. 1994. Requirement of vascular integrin alpha v beta 3 for angiogenesis. Science. 264:569-571.

11. Carron, C.P., et al. 1998. A peptidomimetic antagonist of the integrin $\alpha v \beta 3$ inhibits Leydig cell tumor growth and development of hypercalcemia of malignancy. Cancer Res. 58:1930-1955.

12. Christofidou-Solomidou, M., Bridges, M., Murphy, G., Albelda, S., and DeLisser, H.M. 1997. Expression and function of endothelial cell $\alpha_{v}$ integrin receptors in wound-induced human angiogenesis in human skin/SCID mice chimeras. Am. J. Path. 151:975-983.

13. Drake, C.J., Cheresh, D.A., and Little, C.D. 1995. An antagonist of integrin $\alpha v \beta 3$ prevents maturation of blood vessels during embryonic neovascularization. J. Cell Sci. 108:2655-2661.

14. Friedlander, M., et al. 1996. Involvement of integrin $\alpha v \beta 3$ and $\alpha v \beta 5$ in ocular neovascular diseases. Proc. Natl. Acad. Sci. USA. 93:9764-9769.

15. Varner, J.A., Nakata, M.T., Jordan, R.E., and Coller, B.S. 1999. Inhibition of angiogenesis and tumor growth by murine 7E3, the parent antibody of c7E3 Fab (abciximab; ReoPro ${ }^{\mathrm{TM}}$ ). Angiogenesis. 3:53-61.

16. Bader, B.L., Rayburn, H., Crowley, D., and Hynes, R.O. 1998. Extensive vasculogenesis, angiogenesis, and organogenesis precede lethality in mice lack- ing all $\alpha_{v}$ integrins. Cell. 95:507-520.

17. Kim, S., Bell, K., Mousa, S., and Varner, J.A. 2000. Regulation of angiogenesis in vivo by ligation of integrin $\alpha 5 \beta 1$ with the central cell binding domain of fibronectin. Am. J. Path. 156:1345-1362.

18. Kloss, C., et al. 1999. Integrin family of cell adhesion molecules in the injured brain: regulation and cellular localization in the normal and regenerating mouse facial motor nucleus. J. Comp. Neurol. 411:162-178.

19. Kim, S., Harris, M., and Varner, J.A. 2000. Regulation of integrin $\alpha v \beta 3$ mediated endothelial cell migration and angiogenesis by integrin $\alpha 5 \beta 1$ and protein kinase A. J. Biol. Chem. 275:33920-33928.

20. Varner, J.A., Emerson, D.A., and Juliano, R.L. 1995. Integrin $\alpha 5 \beta 1$ expression negatively regulates cell growth: reversal by attachment to fibronectin. Mol. Biol. Cell. 6:725-740.

21. Giancotti, F.G., and Ruoslahti, E. 1990. Elevated levels of alpha 5 beta 1 fibronectin receptor suppress the transformed phenotype of Chinese hamster ovary cells. Cell. 60:849-859.

22. Plath, T., et al. 2000. A novel function for the tumor suppressor p16 INK4a: induction of anoikis via upregulation of the $\alpha 5 \beta 1$ fibronectin receptor. J. Cell. Biol. 150:1467-1477.

23. George, E. L., Georges, E.N., Patel-King, R.S., Rayburn, H., and Hynes, R.O. 1993. Defects in mesodermal migration and vascular development in fibronectin-deficient mice. Development. 119:1079-1091.

24. Yang, J.T., Rayburn, H., and Hynes, R.O. 1993. Embryonic mesodermal defects in $\alpha 5$ integrin-deficient mice. Development. 119:1093-1105.

25. Goh, K.L., Yang, J.T., and Hynes, R.O. 1997. Mesodermal defects and cranial neural crest apoptosis in $\alpha 5$ integrin-null embryos. Development. 124:4309-4319.

26. Yatohgo, T., Izumi, M., Kashiwagi, H., and Hayashi, M. 1988. Novel purification of vitronectin from human plasma by heparin affinity chromatography. Cell Struct. Funct. 13:281-292.

27. Herrmann, M., et al. 1994. A rapid and simple method for the isolation of apoptotic DNA fragments. Nucleic Acids Res. 22:5506-5507.

28. Dormond, O., Foletti, A., Paroz, C., and Rüegg, C. 2001. NSAIDs inhibit alpha $V$ beta 3 integrin-mediated and Cdc42/Rac-dependent endothelialcell spreading, migration and angiogenesis. Nat. Med. 7:1041-1047.

29. Raynal, P., and Pollard, H. B. 1994. Annexins: the problem of assessing the biological role for a gene family of multifunctional calcium and phospholipid-binding proteins. Biochem. Biophys. Acta. 1197:63-93.

30. Nicholson, D.W., et al. 1995. Identification and inhibition of the ICE/CED3 protease necessary for mammalian apoptosis. Nature. 376:37-43.

31. Partel, T., Gores, G.J., and Kaufmann, S.H. 1996. The role of protease during apoptosis. FASEB J. 10:587-597.

32. Leaveley, D.I., Schwartz, M.A., Rosenfeld, M., and Cheresh, D.A. 1993. Integrin $\beta 1$ and $\beta 3$-mediated endothelial cell migration is triggered through distinct mechanisms. J. Cell Biol. 121:163-170.

33. Johnson, A.L., and Bridgham, J.T. 2000. Caspase- 3 and -6 expression and enzyme activity in hen granulosa cells. Biol. Reprod. 62:589-598.

34. Montgomery, A.M.P, Reisfeld, R.A., and Cheresh, D.A. 1994. Integrin $\alpha v \beta 3$ rescues melanoma from apoptosis in a three-dimensional dermal collagen. Proc. Natl. Acad. Sci. USA. 91:8856-8860.

35. Ashkenazi, A., and Dixit, V.M. 1998. Death receptors: signaling and modulation. Science. 281:1305-1308.

36. Thornberry, N., and Lazebik, Y. 1998. Caspases: enemies within. Science. 281:1312-1316.

37. Schwartz, M.A. 1997. Integrins, oncogenes and anchorage independence. J. Cell Biol. 139:575-578.

38. Frisch, S.M., and Francis, H. 1994. Disruption of epithelial cell-matrix interactions induces apoptosis. J. Cell. Biol. 124:619-626.

39. Rytomaa, M., Martins, L.M., and Downward, J. 1999. Involvement of FADD and caspase 8 signaling in detachment induced apoptosis. Curr. Biol. 9:1043-1046.

40. Magnusson, M.K., and Mosher, D.F. 1998. Fibronectin: structure, assembly, and cardiovascular implications. Arterioscler. Thromb. Vasc. Biol. 18:1363-1370.

41. Stupack, D.G., Puente, X.S., Butsaboualoy, S., Storgard, C.M., and Cheresh, D.A. 2001. Apoptosis of adherent cells by recruitment of caspase- 8 to unligated integrins. J. Cell Biol. 155:459-470.

42. Weissinger, E., et al. 1997. Inhibition of Raf-1 kinase by cyclic AMP agonists causes apoptosis of v-abl-transformed cells. Mol. Cell. Biol. 17:3229-3241.

43. D'Angelo, G., Lee, H., and Weiner, R.I. 1997. CAMP-dependent protein kinase inhibits the mitogenic action of vascular endothelial growth factor and fibroblast growth factor in capillary endothelial cells by blocking Raf activation. J. Cell. Biochem. 67:353-366.

44. Srivastava, R.K., et al. 1998. Involvement of microtubules in the regulation of Bcl-2 phosphorylation and apoptosis through cyclic AMP-dependent protein kinase. Mol. Cell. Biol. 18:3509-3517.

45. Zhang, Z., Vuori, K., Reed, J.C., and Ruoslahti, E. 1995. The $\alpha 5 \beta 1$ integrin supports survival of cells on fibronectin and upregulates bcl-2 expression. Mol. Biol. Cell. 6:841-850.

46. Kim, S., Jee, K., Kim, D., Koh, H., and Chung, J. 2001. Cyclic AMP inhibits Akt activity by blocking the membrane localization of PDK1. J. Biol. Chem. 276:12864-12870. 